\title{
Immunofluorescence pattern of Antinuclear Antibody and its Association with Autoantibody profile in Systemic lupus erythematosus
}

\author{
Sadia Sharmin ${ }^{1}$, Sharmeen Ahmed ${ }^{2}$, Humayun Sattar ${ }^{2}$, Md Ruhul Amin Miah ${ }^{2}$, Minhaz Rahim Chowdhury ${ }^{3}$ and \\ M Masudul Hassan ${ }^{4}$ \\ ${ }^{1}$ Assistant Professor, Department of Microbiology, Anwer Khan Modern Medical College, ${ }^{2}$ Professor, Department of Microbiology and Immunology, \\ BSMMU $^{3}$ Professor, Department of Rheumatology, BSMMU ${ }^{4}$ Medical officer, Department of Rheumatology, BSMMU
}

\begin{abstract}
:
Background: Antinuclear antibody (ANA) is useful in the diagnosis of systemic lupus erythematosus (SLE). Association of specific autoantibodies with the immunofluorescence pattern of ANA in SLE as noted in Western literature has been taken as reference in all over the world. However, in Bangladesh such research work or data correlating the autoantibodies and their ANA patterns is inadequate. Objective: To identify an association between immunofluorescence patterns of antinuclear antibody on HEp-2 cell and more specific antinuclear reactivities (e.g. anti-dsDNA and anti-extractable nuclear antigen) in the serum samples of SLE patients. Methods: Serum samples of 37 SLE patients who were diagnosed by ARA (American Rheumatism Association) classification criteria and laboratory tests, attending at lupus clinic of Bangabandhu Sheikh Mujib Medical University (BSMMU) during the study period of six months were subjected for ANA testing by Indirect Immunofluorescence (IIF) on HEp-2 cell, anti-dsDNA by ELISA and anti- extractable nuclear antigen (anti-ENA) by Dot Immunoblot. Dot blot strips were tested for anti-Sm, anti-RNP, anti-SSA/Ro, and anti-SSB/La. Results: Out of 37 SLE patients $32(86.5 \%)$ cases were ANA positive by IIF on HEp- 2 cell. ANA positive sera exhibited three fluorescence patterns such as speckled (43.7\%), peripheral (34.3\%) and homogenous pattern (21.8\%). Peripheral pattern $(100 \%)$ was strongly associated with anti-dsDNA $(\mathrm{p}<0.05)$ and homogenous pattern $(85.7 \%)$ was also predominantly associated with anti-dsDNA $(\mathrm{p}<0.05)$. Speckled pattern $(85.6 \%)$ was significantly associated with anti-ENA $(\mathrm{p}<0.05)$. Anti-dsDNA was positive in $75 \%$ of SLE cases and majority $(45.8 \%)$ of which showed peripheral pattern whereas anti-ENA was positive in $48.6 \%$ cases and majority ( $70.5 \%$ ) of which showed speckled pattern. The most commonly identified antinuclear autoreactivity was directed towards anti-RNP $(22.2 \%)$ then anti-Sm (16.6\%), anti-SSA (16.6\%) and anti-SSB (11.1\%). Multiple anti-ENA reactivities were identified in 33.3\% cases. Conclusion: Peripheral and homogenous pattern is strongly associated with anti-dsDNA therefore may be predicted that patients have active SLE and speckled pattern may predict anti-ENA (specially ribonucleoprotiens). Thus, ANA-IIF method may suffice and probably reduce the expense of detailed immunological work-up with minimal loss in diagnostic accuracy.
\end{abstract}

Key words: Immunofluorescence antibody, SLE

[BSMMU J 2013; 6 (2) : 141-145]

\section{Introduction :}

Systemic lupus erythematosus (SLE) is an autoimmune disease characterized by the production of various pathological autoantibodies directed against chromatin components and extractable nuclear antigens including double stranded DNA (dsDNA), histones, and most frequently with four groups of RNA binding proteins namely $\mathrm{Sm}$,

Address for Correspondence: Dr, Sadia Sharmin Assistant Professor, Microbiology Anwer Khan Modern Medical College Dhanmondi, Dhaka Email-mhbipul2000@yahoo.com Mobile no-01714295104
U1RNP, SSA/Ro and SSB/La, ${ }^{1,2}$ Indirect immunofluorescence (IIF) on HEp-2 cell is the most commonly used technique for determination of antinuclear antibodies (ANA) because it is highly sensitive with good specificity'. Alternative methods such as ELISA are more likely to cause false positive results. ANA detected in the IIF results yield distinctive staining patterns in the nucleus or cytoplasm of the reagent cells used to perform the test. These staining patterns offer specific clues as to which particular antinuclear antibody or antibodies may be present but does not allow precise identification of these 
autoantibodies. For that purpose, additional testing is required for the detection of specific autoantibodies ${ }^{4,5}$. There is neither research work nor any data available correlating the autoantibodies and their ANA patterns in Bangladesh.

In this study, samples of SLE patients were analyzed for ANA testing by IIF method and further processed for identification of specific autoantibodies by ELISA (antidsDNA) and Dot immunoblot (anti-ENA). The results were correlated with one another to establish any definite link between the fluorescence pattern and specific autoantibody reactivities. If a definite correlation is found between the ANA patterns and immune profile, one could use ANA-IIF fluorescence patterns to predict the presence of autoantibodies to diagnose SLE. This would economize on the cost of laboratory investigations.

\section{Methods :}

A total of 37 diagnosed SLE patients, attending at lupus clinic in BSMMU during the study period of May, 2010 to October, 2010; were enrolled in this cross sectional study. Blood $(5 \mathrm{ml})$ was drawn from SLE patient and sera were separated from the clotted blood samples of all SLE patients by centrifugation. Sera were stored at $4^{\circ} \mathrm{c}$ if testing was planned within 72 hours or at $-20^{\circ} \mathrm{c}$ for testing after three days (without freezing and thawing). Each of the serum samples was subjected for ANA testing by Indirect Immunofluorescence on HEp-2 cell, Dot immunoblot for anti-ENA and ELISA for anti-dsDNA.

Indirect immunofluorescence was done by a commercially available kit. Serum diluted $1 / 40$ in phosphate buffered saline (PBS) was overlaid onto fixed HEp-2 cell (ALPHADIA, Belgium) for 30 minutes at room temperature. Slides were washed twice for five minutes each with PBS, overlaid with fluorescence labeled conjugate, which is antihuman $\operatorname{IgG}$ heavy and light chain specific and incubated for an additional 30 minutes. After washing twice, a coverslip was placed over the slide, and the slides were read using a fluorescence microscope at 40 power ${ }^{4}$. The main fluorescence patterns seen were speckled, homogeneous, and peripheral.

The Dot immunoblot method is a qualitative assay, which utilizes strips of nitrocellulose on which purified antigens are blotted at prelocated spots. Coated antigens used in this study were Sm, RNP, SSA and SSB. The antigen sources used are bovine and rabbit thymus (SSA and Sm) or calf spleen and rabbit thymus (SSB and Sm/RNP). The test procedure was performed according to directions supplied by the manufacturer (D-tek, Belgium). Test strips were incubated for 10 minutes with a diluted patient serum in a PBS-Tween solution. Subsequently the test strips were washed by gentle agitation in a test tube filled with PBS-Tween for $1 \mathrm{~min}$. After the excess buffer solution was removed with a filter paper, the test strips were incubated with an alkaline phosphatase-Protien A conjugate for $10 \mathrm{~min}$. The test strips were then washed for 1 min by gentle agitation by PBS-Tween. Again excess buffer was removed with filter paper. Finally the test strips were stained with 5-bromo-4-chloro-3-indolylphosphate for $5 \mathrm{~min}$. The reaction was terminated by washing the test strips with deionized water. The strips were then air dried. Only strips on which the positive control position was stained as a clearly marked blue spot were able to be evaluated and used for this study ${ }^{6}$.

Antibody against dsDNA was detected by commercially available ELISA kit (Orgentec, Germany). Micro wells were pre-coated with calf thymus dsDNA antigen. The calibrators, controls, and diluted patient samples were added to the wells and autoantibodies recognizing the dsDNA antigen bind during the first incubation. After washing the wells to remove all unbound proteins, purified peroxidase labeled rabbit anti-human IgG conjugate was added. The conjugate binded to the captured human autoantibody and the excess unbound conjugate was removed by a further wash step. The bound conjugate was visualized by TMB substrate which gives a blue reaction product, the intensity of which is proportional to the concentration of autoantibody in the sample. Phosphoric acid was added to each well to stop the reaction. This produces a yellow end point colour, which was read at $450 \mathrm{~nm}$.

Data analysis was performed using SPSS PC version 10 . A chi-square test was used to identify association. A p value $<0.05$ was considered significant.

\section{Results :}

In this cross sectional study, 37 samples of SLE patients 
were analyzed who were diagnosed by ARA (American Rheumatism Association) criteria. We collected serum samples of various stages of SLE patients where autoantibodies were fluctuated by the disease activity. The study tried to find out the correlation of ANA fluorescence pattern with specific autoantibodies. Among these samples, $32(86.5 \%)$ were ANA positive by IIF method. Three patterns of nuclear fluorescence were noted: the homogenous pattern, in which the entire nucleus fluoresced (Fig.1); the peripheral pattern, in which the fluorescence is located along the rim of nucleus (Fig.2); and the speckled pattern in which the fluorescence is localized as discrete spots in the nucleus (Fig.3). In these fluorescence positive samples, speckled pattern was the most common pattern seen in $43.7 \%$ cases, followed by peripheral pattern $34.3 \%$ and homogenous pattern $21.8 \%$.

In this study, Anti-dsDNA was found to be positive in $75 \%$ cases showing peripheral pattern was the most common pattern (Table-I). Peripheral and homogenous pattern in anti-dsDNA positive samples was significant $(\mathrm{p}<0.05)$ (Table-II). Anti-ENA was positive in $48.6 \%$ cases and showing speckled pattern was the most common pattern (Table-I). Speckled pattern was significantly associated with anti-ENA positive samples $(\mathrm{p}<0.05)$ (Table-II). The most commonly identified antinuclear autoreactivity was directed towards anti-RNP $(22.2 \%)$ then anti-Sm (16.6\%), anti-SSA (16.6\%) and anti-SSB $(11.1 \%)$. Multiple anti-ENA reactivities were identified in $33.3 \%$ cases.

ANA-IIF negativity was observed in $5(13.5 \%)$ of the total 37 samples under study. Of these, $4(80 \%)$ exhibited positivity with anti-dsDNA. There was a single case in the entire study that exhibited strong positivity for SSA/Ro but negative for ANA-IIF.
Table-I

Autoantibodies \& fluorescence staining pattern among SLE Patient $(N=32)$

\begin{tabular}{lcc}
\hline IIF Pattern & \multicolumn{2}{c}{ Positive autoantibody } \\
\cline { 2 - 3 } & Anti-dsDNA & Anti-ENA \\
& $\mathrm{n}(\%)$ & $\mathrm{n}(\%)$ \\
\hline Homogenous & $6(25.0)$ & $1(5.88)$ \\
Speckled & $7(29.16)$ & $12(70.58)$ \\
Peripheral & $11(45.83)$ & $4(23.53)$ \\
Total & $24(100)$ & $17(100)$ \\
\hline
\end{tabular}

\section{Table-II}

Probabilities of detecting anti-dsDNA and anti-ENA antibodies according to fluorescence staining pattern in

\section{SLE patients}

\begin{tabular}{lccc}
\hline & \multicolumn{3}{c}{ Fluorescence Pattern } \\
\cline { 2 - 4 } Specific & $\begin{array}{c}\text { Homogenous } \\
\text { autoantibodies }\end{array}$ & $\begin{array}{c}\text { Speckled } \\
(\mathrm{n}=7)\end{array}$ & $\begin{array}{c}\text { Peripheral } \\
(\mathrm{n}=14)\end{array}$ \\
\hline Anti-dsDNA & $6(85.7 \%)$ & $7(50 \%)$ & $11(100 \%)$ \\
Anti-RNP & 0 & $4(28.5 \%)$ & 0 \\
Anti-SM & $1(14.2 \%)$ & $1(7.1 \%)$ & $1(9.0 \%)$ \\
Anti-SSA & 0 & $2(14.2 \%)$ & 0 \\
Anti-SSB & 0 & $2(14.2 \%)$ & 0 \\
Multiple anti-ENA & 0 & $3(21.4 \%)$ & $3(27.2 \%)$ \\
\hline
\end{tabular}

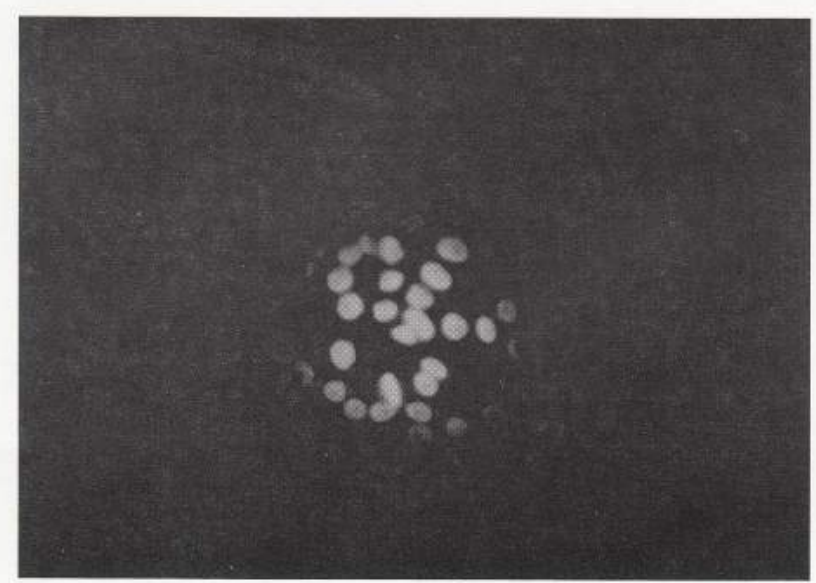

Fig-1: Homogenous pattern 


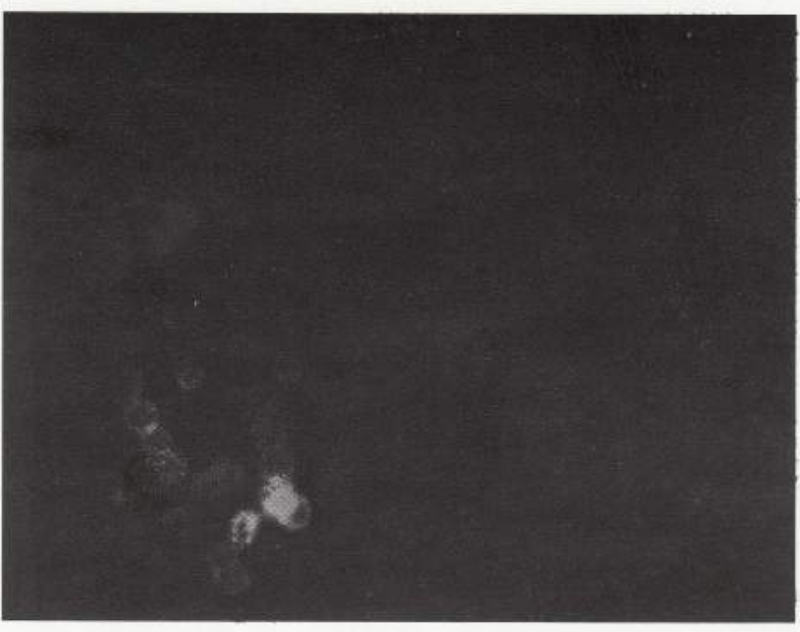

Fig-2: Peripheral pattern

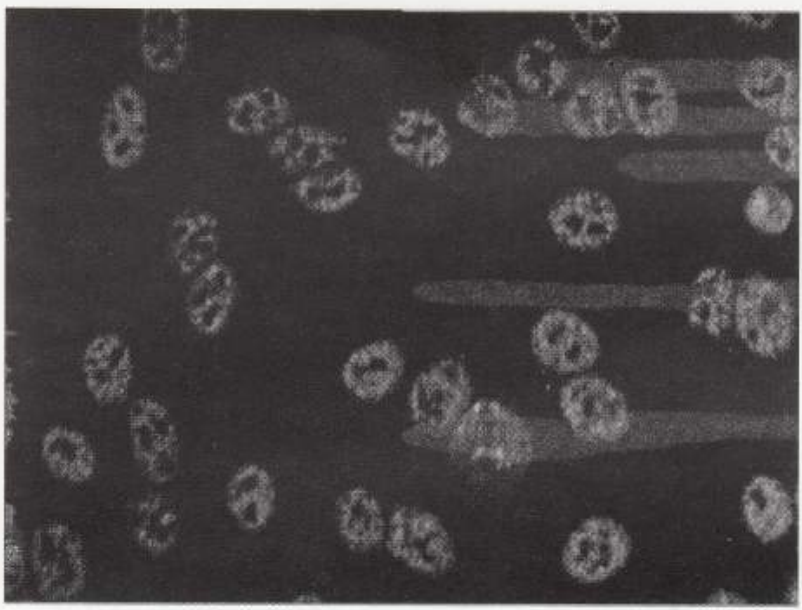

Fig-3: Speckled pattern

\section{Discussion:}

Antinuclear antibody testing is usually the first step in the immunologic diagnosis of SLE. Indirect Immunofluorescence on HEp-2 cell is the standard approach for detecting ANAs, and the staining patterns depend on the location of the target antigen. These patterns correspond to the presence of autoantibodies against different nuclear antigens $^{7,8}$. We report here on the probability of identifying specific antinuclear reactivities (anti-dsDNA and anti-ENA antibodies) in ANA positive serum samples, using ELISA and Dot blot respectively. The fluorescence pattern itself does not disclose the fine reactivity of the detected antibodies (except for anticentromere antibodies). However, the fluorescence pattern may be indicative of the type of reactivities responsible for the IIF staining. In this study, anti-dsDNA antibodies were commonly identified in serum samples with homogenous and periph- eral ANA staining. Anti-ENA antibodies were more randomly distributed between different fluorescence patterns. It should be noted that the fluorescence pattern may result from the simultrneous occurrence of different ANA.

In this study, Indirect Immunofluorescence on HEp-2 cell reveled three staining patterns in serum of SLE patients: most commonly speckled pattern $(43.75 \%)$ followed by peripheral $(34.3 \%)$ and homogenous pattern $(21.8 \%)$ whereas in New York Gonzalez and Rothdfield reported that the homogenous and peripheral patterns were seen most frequently in serum from patients with systemic lupus erythematosus and only a few produced a speckled pattern $^{9}$

Speckled pattern, frequently identified ANA pattern in this study showed an association with Sm, RNP, SSA/Ro or SSB/La. Thus, with a speckled pattern one could project further that the serum had antibodies against antiSm, RNP, SSA or SSB. In USA Mutasim and Adams also reported similar association between speckled pattern and various ribonucleoprotien in their study ${ }^{10}$. The next common peripheral pattern observed in this study showed an association with anti-dsDNA with variable intensities of SSA, SSB and RNP/Sm. Thus, one could predict the presence of anti-dsDNA in $100 \%$ of cases with speckled pattern. White and Robbins also described in their study that the peripheral pattern of nuclear staining is characteristically associated with the presence of serum antibodies directed against DNA ${ }^{11}$. Weitzman and Walker had similar observation $^{12}$. Homogenous pattern was strongly associated with antibodies to dsDNA which is consistent with the findings of Peene et $\mathrm{al}^{4}$. Sebastian et al conducted another study and observed similarly ${ }^{13}$.

Positive ANA-IIF with negative Dot immunoblot was interestingly noted. A positive result with ANA-IIF, together with negative results in line immunoassay, was also noted earlier and attributed to the presence of anti-dsDNA antibodies. Vos et al have found ANA positive samples negative with line immunoassay but found positive for anti-dsDNA antibodies ${ }^{14}$.

In this study, 4 samples were ANA-IIF negative. However, they had positive anti-dsDNA. This could be 
explained by that antibodies tend to rise during flares of disease ${ }^{5}$.

There was only one case which was positive for anti-ENA by Dot immunoblot, was negative by ANA-IIF. This serum showed anti- SSA positivity. Though a single case is insufficient to give any explanation, Hoffman et al explained by the fact that line immunoassay is more sensitive for the detection of SSA/Ro-52 than ANA-IIF even when Hep-2000 cells are used as SSA is a cytoplasmic antigen $^{15}$.

In conclusion, Immunofluorescence pattern could predict the presence of certain specific antibodies in the sera. These correlations are of relevance for the diagnosis of disease more specifically. Dot immunoblot may be considered as a special investigation particularly for clinically suspected cases with unusual presentation or patients with overlap syndrome. This test may be also useful in suspected SLE patients in which ANA-IIF either negative or shows cytoplasmic pattern.

\section{Acknowledgement :}

We would like to thank to the department of Microbiology and Immunology, and department of Rheumatology of BSMMU for their support.

\section{References :}

1. Sallai K, Nagy E, Derfalvy B, Müzes G and Gergely P. Antinucleosome Antibodies and Decreased Deoxyribonuclease Activity in Sera of Patients with Systemic Lupus Erythematosus. Clinical and diagnostic laboratory immunology. 2005; 12: 56-9.

2. Maddison PJ. Rheumatic diseases associated with antinuclear antibodies. Arthritis Research Campaign. 2001.
3. Tozzoli R, Bizzaro N, Tonutti E, Villalta D, Bassetti D, Manoni F, et at P. Guidelines for the Laboratory Use of Autoantibody Tests in the Diagnosis and Monitoring of Autoimmune Rheumatic Diseases. Am J Clin Pathol. 2002; 117:316-24.

4. Peene I, Meheus L, Veys EM, Keyser FDe. Detection and identification of antinuclear antibodies (ANA) in a large and consecutive cohort of serum samples referred for ANA testing. Ann Rheum Dis. 2001; 60:1131-36.

5. Moore E. Diagnosing Lupus and Related Connective Tissue Disorders. Laboratory diagnosis of Autoimmune Disease. 2002.

6. Ermens AAM, Bayens AJM, van Gemert ACM, van Duijnhoven LP. Simple dot-blot method evaluated for detection of antibodies against extractable nuclear antigens. Clin Chem 1997; 12:2420-22.

7. Arbuckle MR, McClain MT, Rubertone M., et al. Development of autoantibodies before the clinical onset of systemic lupus erythematosus. N Engl J Med. 2003; 349:1526-33.

8. Emlen W, O'Neill L. Clinical significance of antinuclear antibodies: Comparison of detection with immunofluorescence and enzymelinked immunosorbent assays. Arthritis Rheum. 1997; 40:1612-18.

9. Gonzalez EN and Rothfield NF. Immunoglobulin class and pattern of nuclear fluorescence in systemic lupus erythematosus. New Eng J Med.1966;274:1333-38.

10. Mutasim DF and Adams BB. A practical guide for serologic evaluation of autoimmune connective tissue diseases. J Am Acad Dermatol. $2000 ; 42: 159-74$.

11. White RH and Robbins DL. Clinical Significance and Interpretation of Antinuclear Antibodies. West J Med. 1987; 147:210-13.

12. Weitzman $R \mathrm{~J}$ and Walker $\mathrm{S} E$. Relation of titred peripheral pattern ANA to anti-DNA and disease activity in systemic lupus erythematosus. Annals of the Rheumatic Diseases. 1977; 36: 44-9.

13. Sebastian W, Roy A, Kini U, Mullick S. Correlation of antinuclear antibody immunofluorescence patterns with immune profile using line immunoassay in the Indian scenario. Indian J Pathol Microbiol. 2010; 53:427-32.

14. Vos PA, Bast EJ, Derksen RH. Cost-effective detection of non-antidouble-stranded DNA antinuclear antibody specificities in daily clinical practice. Rheumatology (Oxford). 2006; 45:629-35

15. Hoffman IE, Peene I, Veys EM, De Keyser F. Detection of specific antinuclear reactivities in patients with negative anti-nuclear antibody immunofluorescence screening tests. Clin Chem. 2002; 48:2171-6. 